\title{
The Study on the Membership Function Shape of Fuzzy Controller Optimization
}

\author{
Tao $\mathrm{Wu}^{1, \mathrm{a}}$, Zhenhai $\mathrm{Gao}^{1, \mathrm{~b}}$ and Yang You ${ }^{1, \mathrm{c}}$ \\ ${ }^{1}$ State Key Laboratory of Automotive Simulation and Control, Jilin University, Changchun, China \\ a32151020@163.com, b1264415075@163.com, 'Yy08@163.com
}

\begin{abstract}
Keywords: Adaptive Cruise Control; Fuzzy Self-Tuning Controller; Membership function; Particle Swarm Optimization.
\end{abstract}

\begin{abstract}
Since it is difficult to determine a fuzzy controller parameters, which need to match the test car's complex nonlinear dynamic characteristics. Based on particle swarm optimization algorithm, a fuzzy self-tuning controller parameters optimization method is developed. The controller is reconstructed by using particle swarm optimization, which can randomly chosen values in the value range of membership function's shape point in many times. And to keep track of the objective function as best, the membership function shape optimization problem of fuzzy self-tuning controller is solved. The simulation results show that the optimized controller has good control performance, which can get controller parameters by using particle swarm optimization method.
\end{abstract}

\section{Introduction}

Adaptive Cruise Control (ACC) is a common and well known automotive driver assistance system. Relying on the radar sensor, it can automatically adjust speed in order to maintain a safe headway distance between front vehicles in the same lane[1].

Recently many ACC control algorithms have been presented. The initial ACC control algorithm major uses the equivalent linearization method to represent the nonlinear systems. Based on the linear reference model, a linear control strategy, such as PID, is designed[2]. This method can get better control accuracy at low speed because the vehicle dynamic characteristic in the linear region. But it becomes very inaccurate with the speed rising. In order to better solve the vehicle strongly nonlinear dynamics problem, the researchers presents fuzzy control and intelligent control algorithm for ACC[3]. However, it is very difficult to establish a good dynamic and steady state characteristics of fuzzy controller, because different test vehicles own different nonlinear and complex characteristics, and presently still lack a simple and efficient design and parameters adjustment method.

Particle swarm optimization algorithm derived from the study of birds' predatory behavior, which is a kind of stochastic optimization algorithm based on swarm intelligence[4].

In this paper, since it is difficult to determine the fuzzy self-tuning controller parameters for adaptive cruise control, which need to match the test vehicle's complex nonlinear dynamic characteristics, so the membership functions of fuzzy self-tuning controller optimization method is developed based on particle swarm optimization algorithm, which can effectively reduce work on matching the adaptive cruise control system and ensure good control performance for test vehicle.

\section{Imitated the driver behavior of ACC}

Through the study of driver handling behavior characteristics, paper [5] developed a driver model, which simulates driver behaviour. So on basis of driver steady preview and dynamic calibration hypothesis, the ACC system is established and shown in figure 1. It can be divided into information identification, preview decision-making and control correction. In paper [6], based on the classical fuzzy controller, a novel fuzzy self-tuning control algorithm of ACC is established and this controller's parameters can be tuned on-line based on the evaluation indexes that can describe how the driver consider the quality of dynamical characteristic of vehicle longitudinal dynamics. The control algorithm block diagram is shown in figure 2 . 


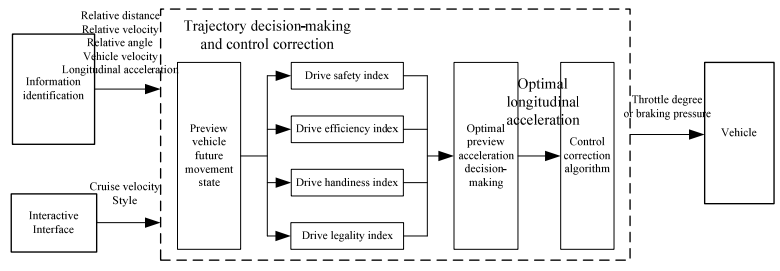

Fig. 1 ACC system flow diagram

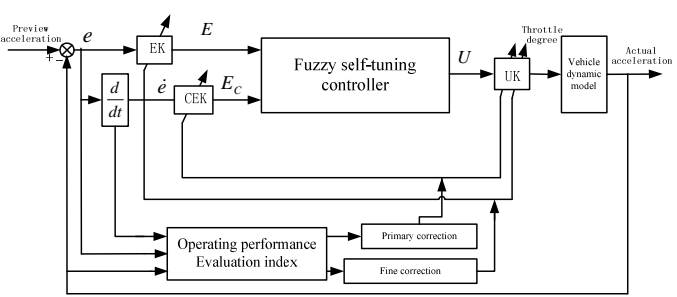

Fig. 2 The fuzzy controller block diagram

The controller has two inputs and one output. The input parameters are the error and the error rate between the optimal preview acceleration and the actual acceleration and the output parameter is the increment of accelerator or brake pedal. $E K$ is the quality factor of accelerator error $e, C E K$ is the quality factor accelerator error rate $\dot{e}$ and $U K$ is the quality factor of pedal increment $u$. Through analysis, the initial value of three controller parameters is selected, which are 4, 4 and 6.

\section{Optimization of membership functions of the fuzzy self-tuning controller}

Since membership functions of the controller has important impact on controller performance, and it is very difficult to determine the membership functions shape. So optimization of membership functions of the controller using particle swarm optimization method is developed, which can optimize membership functions shape of the controller inputs to simplify the controller design.

ACC algorithm simulation environment model. Carsim and Simulink joint simulation program of ACC algorithm is built and shown in figure 3. Carsim module presents vehicle dynamic model, Decision-making module and control correction module are established in simulink. And the objective function of particle swarm optimization algorithm is the minimum error between ideal acceleration and actual acceleration. The entire optimization process flow chart is shown in figure 4 .

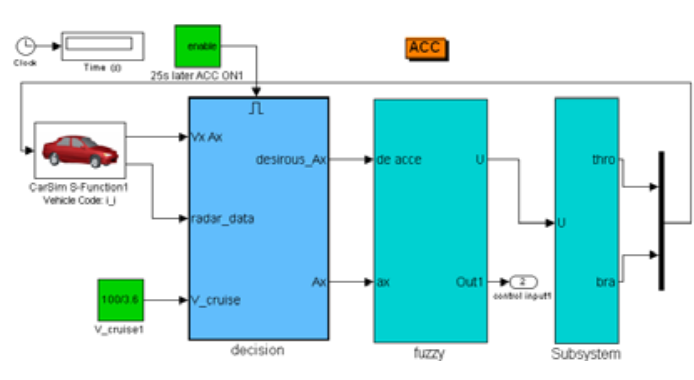

Fig. 3 The ACC system program

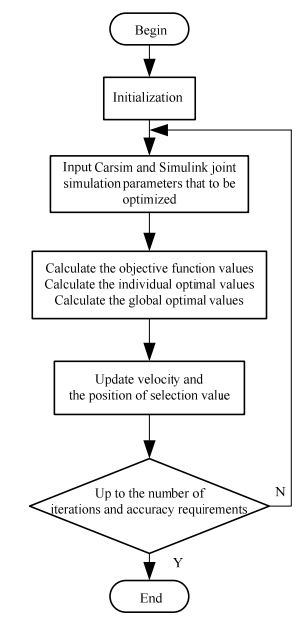

Fig. 4 The optimization process flow chart

Algorithm parameters configuration. Parameter settings are as follows in the particle swarm optimization algorithm. The maximum number of iterations is set to 800 , and the number of initial particles is 24 . According to the membership function shape, the number of optimized parameter is 20 , so the initial matrix is the 24 row and the 20 column. Acceleration parameter is set to 2 . The terminal threshold of algorithm is 1e-25. The optimization parameters range can appropriate selection based on their original value.

Typical conditions of optimization process. Taking into account the common level of speed and typical operating conditions of the ACC system, optimized operating conditions are set as follows. A constant speed of the front vehicle is $80 \mathrm{~km} / \mathrm{h}$. And the hosting vehicle with a constant speed $100 \mathrm{~km} / \mathrm{h}$ gradually close to the front vehicle. Then the radar detects the front vehicle, the hosting vehicle slows down and has the same speed with the front vehicle into following state. The front vehicle cut in the other lane in 40s, so the hosting vehicle accelerates to the cruise speed, which is set to $100 \mathrm{~km} / \mathrm{h}$. The simulation time is 80 s. 
The optimized results and analysis. The optimized membership function shapes of the acceleration error $e$ and the acceleration error rate $\dot{e}$ are shown in figure 5, 6 .

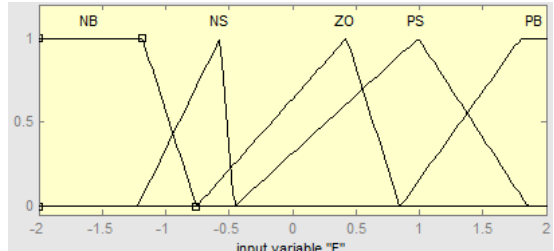

Fig. 5 Optimized membership function shape $e$

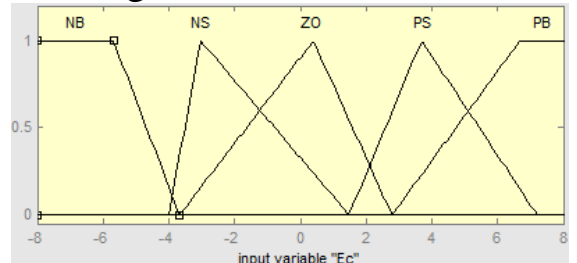

Fig. 6 Optimized membership function shape $\dot{e}$

\section{Simulation}

Low and high speed level of typical operating conditions are adopted to verify the performance of the optimized controller. Simulation results of two controllers are compared in before and after optimization.

Simulation of Low-speed operating conditions. In initial, there is a front vehicle in the same lane with the ACC vehicle, but the distance between the two vehicles go beyond the scope of radar detection, which is $500 \mathrm{~m}$. The initial speed of the hosting vehicle is $40 \mathrm{~km} / \mathrm{h}$, and cruise speed is set to $60 \mathrm{~km} / \mathrm{h}$. The front vehicle with a constant speed is $30 \mathrm{~km} / \mathrm{h}$ in initial, and begins to accelerate in $70 \mathrm{~s}$ that constantly accelerate from $30 \mathrm{~km} / \mathrm{h}$ to $60 \mathrm{~km} / \mathrm{h}$ in the $150 \mathrm{~s}$. Then constantly decelerate to $50 \mathrm{~km} / \mathrm{h}$ in the $150 \mathrm{~s}$ after driving with constant speed $60 \mathrm{~km} / \mathrm{h}$ in the $100 \mathrm{~s}$. Finally, the front vehicle travels at constant speed $50 \mathrm{~km} / \mathrm{h}$. The simulation results of ACC system controlling the vehicle are shown in figure 7-8 under this condition.

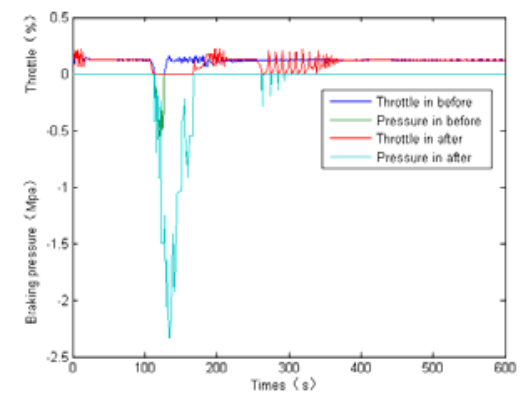

Fig. 7 Comparison curve of throttle degree and braking pressure

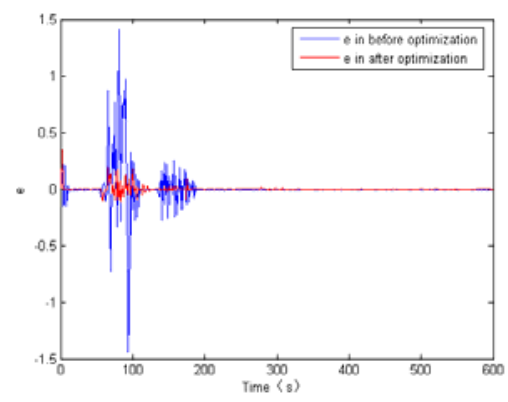

Fig. 8 Comparison curve e in Low-speed operating conditions

It can be seen from figure 7, both before and after optimization of the controller can achieve the effect and purpose of speed control in the low-speed operating condition. However, the optimized curves of throttle degree and brake pressure are more stable and less oscillatory, and smoother in speed control in the process of stable following and cruise control. And the optimized curves of throttle degree and brake pressure fluctuates greater, and brake and throttle control frequently switch to achieve good following feature when the hosting vehicle is free travel and follows the front vehicle with changing speed. It can be seen from figure 8 , the optimized controller controls more stable.

Simulation of high-speed operating condition. In initial, there is a front vehicle in the same lane with the ACC vehicle, but the distance is $1000 \mathrm{~m}$. The initial speed of the hosting vehicle is $100 \mathrm{~km} / \mathrm{h}$, and cruise speed is set to $150 \mathrm{~km} / \mathrm{h}$. The front vehicle with a constant speed is $110 \mathrm{~km} / \mathrm{h}$ in initial, and begins to accelerate in $70 \mathrm{~s}$ that constantly accelerate from $110 \mathrm{~km} / \mathrm{h}$ to $150 \mathrm{~km} / \mathrm{h}$ in the $150 \mathrm{~s}$. Then constantly decelerate to $140 \mathrm{~km} / \mathrm{h}$ in the 150 s after driving with constant speed $150 \mathrm{~km} / \mathrm{h}$ in the $100 \mathrm{~s}$. Finally, the front vehicle travels at constant speed $140 \mathrm{~km} / \mathrm{h}$. The simulation results are shown in figure 9-10. It also can be seen the optimized controller controls more stable is better. 


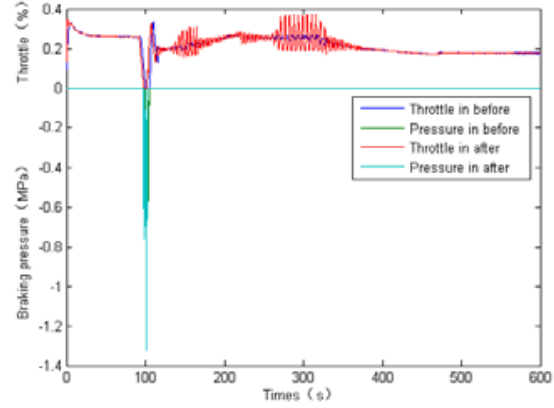

Fig. 9 Comparison curve of throttle degree and braking pressure

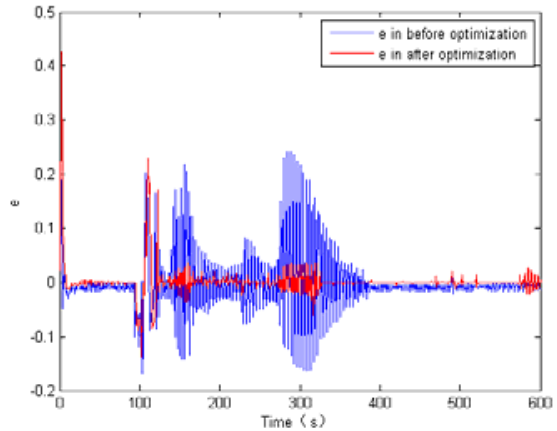

Fig. 10 Comparison curve e in high-speed operating conditions

\section{Conclusion}

In this paper, based on particle swarm optimization algorithm, a fuzzy self-tuning controller parameters optimization method is developed, which can solve the problem that is difficult to determine the controller parameters, which need to match the test vehicle's complex nonlinear dynamic characteristics. Through particle swarm optimization method, the triangle membership function shape of the fuzzy self-tuning controller's inputs is designed. The simulation results show that the proposed method is effective and the optimized fuzzy self-tuning controller has superior performance.

\section{References}

[1] Brackstone M: Car-following: a historical review[J]. Transportation Research Record(1999), 2(4):181 196.

[2] Kazuo T, Hua O. Wang: Fuzzy Control Systems Design and Analysis: A Linear Matrix Inequality Approach (Wiley Publications, New York 2001).

[3] Guan H, Wang J W, Gao Z H: An Acc Control Algorithm Based on Driver Behavior Model[J]. Automotive Engineering, 2004051.

[4] Shi Y, Eberhart R: A modified particle swarm optimizer[A]. Proceedings of the 1998 IEEE.

[5] Guan H, Gao Z H: A Driver Direction Control Model and its Application in the Simulation of Driver-Vehicle-Road Closed-Loop System[J]. SAE paper, 2000-01-2184.

[6] Gao Z H, Guo J: A Fuzzy On-Line Self-Tuning Control Algorithm for Vehicle Adaptive Cruise Control System with the Simulation of Driver Behavior[J]. SAE paper, 2009-01-1481. 